\title{
Qualitätssicherung - auch aus der Sicht der Patienten
}

\section{O. Frank}

\section{Einleitung}

In der fortwährend aktuellen Diskussion gesundheitspolitischer Themen nimmt das Qualitätsmanagement im akutstationären Bereich einen sehr grossen Stellenwert ein. Die Bestrebungen, medizinische Qualität zu sichern und zu verbessern, sind ungebrochen. Bis vor wenigen Jahren war in den Schweizer Spitälern noch kaum die Rede von Qualitätsmanagement. Erst durch den ständig wachsenden Kostendruck entstand die Notwendigkeit, qualitätssichernde Massnahmen zu ergreifen, welche trotz verminderter Ressourcen eine gleichbleibende oder sogar verbesserte Qualität gewährleisten sollen. Qualitätsmanagement - also nur eine Modeerscheinung?

\section{Auswirkungen gesetzlicher Vorgaben}

Nicht nur das Inkrafttreten des Bundesgesetzes über die Krankenversicherung (KVG) [1], sondern auch die ausführenden Verordnungen zum Krankenversicherungsgesetz (KVV) [2] zwingen die Leistungserbringer, Verantwortung für die Qualitätssicherung und deren Nachweis zu übernehmen.

Verschiedene Institutionen setzen diesbezüglich ihre Qualitätssicherung an unterschiedlichen Grundpfeilern an. Die BfS-Statistik [3] erfasst reine Falldaten aus den Spitälern, Fachgesellschaften erfassen von ihnen eigens ausgewählte klinische Patientendaten und verschiedene Institute die Patientenzufriedenheit.

Gemeinsam erklärtes Ziel aller ist, systematisch Daten zu sammeln und für das Qualitätsmanagement zur Verfügung zu stellen. Oftmals werden dabei nur Statistiken über medizinische Eingriffe und deren Komplikationen erstellt. Die Registrierung reiner Eintritts- und Eingriffsdaten ist jedoch nicht ausreichend, um die Qualität auch aus Patientensicht zu bemessen. Doppelspurigkeiten bei der Datenerfassung verschiedener Fachgesellschaften und Vereinigungen werden dabei ausser acht gelassen.

\section{Der Verein Outcome}

Der Verein Outcome ist seit Januar 2000 operativ tätig. Er ist eine Non-Profit-Organisation und bildet einen Zusammenschluss von Leistungserbringern (Spitäler), Versicherern (Kranken- und Unfallversicherer) und der Kantone (Gesundheitsdirektionen). Die Patientenorganisationen und die zuweisenden Grundversorger (Hausärzte) sind ständige Beiräte. Der Verankerungsvertrag regelt die Zusammenarbeit, die inhaltlichen und organisatorischen Aspekte sowie die Prinzipien der Steuerung zwischen den drei Parteien. Die beteiligten Spitäler verpflichten sich, Outcome-Messungen (Ergebnisqualitätsmessungen) durchzuführen. Die Versicherer und die Gesundheitsdirektionen verpflichten sich im Gegenzug, die Messungen als zusätzliche Leistung zu finanzieren. Damit sind die OutcomeMessungen Teil des Leistungsauftrages der Spitäler.

Das Ziel des Verein Outcome ist es, die Spitäler auf der Basis der Ergebnisqualitätsmessungen in ihrer Qualitätsentwicklung zu fördern und dafür eine datenbasierte Grundlage zur Verfügung zu stellen [4].

\section{Patientenorientierung}

Der Verein Outcome führt patientenorientierte Qualitätsmessungen in den akutsomatischen Spitälern der Kantone Zürich, Bern, Aargau und Solothurn durch. Patientenorientierte Qualitätsarbeit in den Spitälern bedeutet, sich mit Strukturen und Prozessen auseinanderzusetzen, mit dem Ziel, ein gutes Ergebnis für den Patienten zu erreichen. Die entscheidende Frage dabei lautet: Wurden die Leistungen so erbracht, dass sie aus klinischer Sicht und aus Sicht des Patienten gut waren? Die im Gesundheitswesen immer noch vorherrschende biomedizinische Sichtweise, die durch ihre Fokussierung auf körperliche Aspekte von Krankheit wesentlich andere Aspekte im psychischen und sozialen Bereich ausser acht 
lässt, spiegelt sich auch in verschiedenen Fachgesellschaften bei der Erfassung von Qualitätsdaten wider. Es genügt nicht, wenn die Fachexperten ihr eigenes Qualitätsverständnis definieren und dafür entsprechende Daten für das Qualitätsmanagement zur Verfügung stellen [5].

Die Qualität der Leistungen eines Spitals wird beim Verein Outcome nicht nur auf Grund der erreichten medizinischen Kennzahlen erfasst, sondern auch auf Grund der erreichten Verbesserung der Lebensqualität der Patienten. Dabei werden zum einen klinische Patientendaten erhoben (Diagnose- und Therapieparameter, Komplikationen), zum anderen werden die Patienten während oder nach einem Spitalaufenthalt mittels Patientenfragebogen hinsichtlich ihrer Erfahrungen und Erlebnisse befragt. Die Sicht des Patienten und seine konkreten Erfahrungen werden dabei zum Ausgangspunkt von Qualitätsverbesserungen gemacht. Qualitätssicherung macht nur dann einen Sinn, wenn Experten- und Patientensicht gemeinsam in die Qualitätsbemühungen einbezogen werden. Wem sonst, als dem Patienten, sollen die Bemühungen zur Qualitätssicherung nützen?

\section{Diagnosespezifische Patientenfragebogen}

Die Grundlagen für die heutigen Outcome-Messungen sind auf das Spitalreformprojekt LORAS* (1995-1999) der Gesundheitsdirektion des Kantons Zürich zurückzuführen. Darin wurden die Outcome-Messungen als ein Begleitelement zur Einführung der leistungsorientierten Spitalfinanzierung eingeführt und als notwendiges Instrument, flankierend zur neuen Spitalsteuerung, eingesetzt.

$\mathrm{Zu}$ gesundheitspolitisch und ökonomisch relevanten, ausgewählten Erkrankungen wurden Messthemen und entsprechende Messinstrumente entwickelt, die Daten für das Qualitätsmanagement in den Spitälern erfassen. Um die Patientensicht bei bedeutsamen diagnosebezogenen Messungen (Tracermessungen) zu berücksichtigen, wurden in Fokusgruppen spezifische Patientenfragebogen bez. der Lebensqualität oder des Wissens und der Sicherheit der Patienten bei Austritt entwickelt.

Patientenorientierung heisst, dass sich im Rahmen eines therapiekonformen Betreuungsprozesses alle Berufsgruppen daran beteiligen,

Tabelle 1

Messthemenspezifische Patientenfragebogen der Outcome-Messungen.

\begin{tabular}{|c|c|}
\hline Messthema: & Patientenfragebogen fragt nach: \\
\hline $\begin{array}{l}\text { Appendizitis und Verdacht auf Appendizitis behandelt } \\
\text { mittels Appendektomie }\end{array}$ & «Wissen und Sicherheit bei Austritt» \\
\hline Proximale Femurfraktur & ¿Wissen und Sicherheit bei Austritt» \\
\hline Akuter Myokardinfarkt & ¿Wissen und Sicherheit bei Austritt» \\
\hline & $\begin{array}{l}\text { «Lebensqualität nach akutem } \\
\text { Myokardinfarkt nach } 2 \text { Monaten» }\end{array}$ \\
\hline Zerebrovaskulärer Insult & ¿ «Unterstützung und Betreuung bei Austritt» \\
\hline Geburt / Sectio & ¿Wissen und Sicherheit bei Austritt» \\
\hline \multirow[t]{3}{*}{ Benigne Prostatahyperplasie (TURP) } & $\begin{array}{l}\text { «Symptome und Beeinträchtigungsscore } \\
\text { vor Eintritt» }\end{array}$ \\
\hline & 凹 «issen und Sicherheit bei Austritt» \\
\hline & $\begin{array}{l}\text { «Symptome und Beeinträchtigungsscore } \\
4 \text { Monate postoperativ» }\end{array}$ \\
\hline Totalendoprothese bei primärer Coxarthrose & 口 «WOMAC - vor Eintritt, präoperativ» \\
\hline & «Wissen und Sicherheit bei Austritt» \\
\hline & $\square$ «WOMAC - 3 Monate postoperativ» \\
\hline & aWOMAC - 12 Monate postoperativ» \\
\hline $\begin{array}{l}\text { Anästhesiologische Komplikationen und perioperative } \\
\text { Beschwerden? }\end{array}$ & $\begin{array}{l}\text { «Subjektive Beschwerden und Beeinträch- } \\
\text { tigung der Patienten durch die Anästhesie» }\end{array}$ \\
\hline Elektiver Eintritt & • «Zufriedenheit bei elektivem Eintritt» \\
\hline $\begin{array}{l}\text { Emerge - sichere und schnelle Hilfe in der Notfall- } \\
\text { situation? }\end{array}$ & $\begin{array}{l}\text { «Zufriedenheit mit der Notfallbehandlung - } \\
\text { subjektive Einschätzung der Patienten» }\end{array}$ \\
\hline Patientenzufriedenheit & $\begin{array}{l}\text { «Patientenzufriedenheit und Umgang } \\
\text { mit Reklamationen» }\end{array}$ \\
\hline
\end{tabular}


die Erwartungen und Bedürfnisse der Patienten kennenzulernen und zu erfüllen [6]. Ausgang dafür können nur Patientenbefragungen sein, deren Ergebnisse Aussagen darüber ermöglichen, welche speziellen Bedürfnisse die Patienten bei einer bestimmten Erkrankung haben und ob diese während eines stationären Aufenthaltes erfüllt wurden. Patientenorientierung setzt eine für den Patienten verständliche Information, die Beteiligung und den Einbezug des Patienten in den Behandlungsprozess, die Abstimmung der Erwartungen mit dem diagnostisch-therapeutisch und pflegerisch Notwendigen und darauf abgestimmte Prozesse in den Spitälern voraus.

In Benchmarking-Workshops werden die Messergebnisse von Fachexperten der Spitäler analysiert, auffällige Unterschiede benannt und mögliche Ursachen diskutiert. Durch den gezielten Erfahrungsaustausch bez. möglicher Verbesserungsmassnahmen in den Spitälern wird ein Lernen vom Besten ermöglicht.

\section{Umgang mit den Daten}

Die von den Spitälern erfassten Daten werden streng anonym nach definierten Regeln des Vereins Outcome behandelt. Die Messergebnisse aller Spitäler werden in den Auswertungen anonym dargestellt, wobei jedes Spital seine eigenen Messergebnisse und die des Gesamttotals erkennen kann. In den Benchmarking-Workshops kann unter bestimmten Voraussetzungen die Anonymität aufgehoben und damit spitalscharf über Gemeinsamkeiten und Unterschiede der Messergebnisse diskutiert werden. Erfahrungen und das wachsende Vertrauen der Spitäler untereinander, gegenüber den Finanzierern und der Gesundheitsdirektionen zeigen, dass 80\% aller bisherigen Benchmarking-Workshops unter Aufhebung der Anonymität stattfanden.

\section{Fazit}

Qualitätsmanagement ist keine Modeerscheinung, solange die Leistungserbringer ernsthaft an der Qualitätsverbesserung der Leistungen im Sinne des Patienten arbeiten. Integraler und somit unverzichtbarer Bestandteil des Qualitätsmanagements in den Spitälern ist die Verbesserung der Dienstleistungsqualität durch die Nutzung der Patientenerfahrungen. Fachexperten müssen sich auch mit den Qualitätsvorstellungen der Patienten auseinandersetzen. Die $\mathrm{Zu}-$ sammenarbeit und Kooperation verschiedener Fachgesellschaften kann dabei wertvolle Synergien zum Nutzen des Patienten und eines verbesserten Qualitätsmanagements schaffen.

Literatur

1 Krankenversicherungsgesetz (KVG/1995), SR 832.10, Art. 58. www.admin.ch/ch/d/sr/ c832_10.html.

2 Verordnung zum Krankenversicherungsgesetz (KVV/1996), SR 832.102, Art. 77. www.admin.ch/ ch/d/sr/c832_102.html.

3 Bundesamt für Statistik (BfS). Medizinische Statistik der Krankenhäuser. Detailkonzept 1997. www.statistik.admin.ch/stat_ch/ber14/gewe/ detailkonz_ms_5-97_kompl_d.pdf.

4 Eichler K, Hochreutener M-A. Outcome-Messung im Krankenhaus: Wege zur Erhöhung der Datenqualität. Schriftenreihe der SGGP. No. 67.

5 Klemperer D. Patientenorientierung im Gesundheitssystem. Qualität in der Gesundheitsversorgung- Newsletter der GQMG. 2000;7(1):15.

6 Widmer W. Patientenzufriedenheit verbessern aber wie? Managed Care. 2003;7:15. 